\title{
MÉTHODES D'ÉTUDE DE COUCHES MINCES ET D'INTERFACES PAR SPECTROSCOPIE RAMAN
}

\author{
Y. LEVY \\ Institut d'Optique $\left({ }^{*}\right)$, Université Paris XI, 91405 Orsay, France
}

R. DUPEYRAT

Département de Recherches Physiques (*), Université de Paris VI, Paris, France

\begin{abstract}
Résumé. - Nous présentons différentes méthodes destinées à augmenter le flux Raman diffusé par une couche mince ou une tranche superficielle située au voisinage d'une interface.
\end{abstract}

\begin{abstract}
We present here some experimental methods which can amplify the Raman scattering flux of a thin film or a thin slice in the neighbourhood of an interface.
\end{abstract}

1. Introduction. - L'interprétation de spectres Raman et infra-rouge de matériaux permet d'identifier un grand nombre de vibrations caractéristiques et de les attribuer à des conformations spécifiques. Le but de cette publication est de synthétiser quelques méthodes d'obtention de spectres Raman de matériaux se présentant sous forme de couches minces $[1,2]$ ou de tranches superficielles situées au voisinage d'une interface [3]. Nous montrerons que l'une des méthodes permet d'atteindre des spectres Raman de couches superficielles placées au niveau de l'interface séparant deux milieux diélectriques semi-infinis. Les méthodes que nous décrirons ont pour rôle essentiel de créer dans la couche mince ou dans la tranche superficielle une forte densité d'énergie lumineuse [4], indispensable à l'augmentation dans de fortes proportions, du flux Raman diffusé. La comparaison d'un spectre Raman obtenu sur un matériau massif avec celui obtenu à partir de ce même matériau au contact avec un substrat permet également de caractériser certaines modifications de conformation au niveau de l'interface [5].

L'étude de la diffusion Raman sur de très petits volumes de matériau, comme dans le cas des couches minces, pose quelques problèmes expérimentaux difficiles. Comme nous l'avons indiqué plus haut, le principe que nous avons utilisé, consiste à accumuler l'énergie lumineuse à l'intérieur du matériau. Dans la première méthode, la couche est utilisée en tant que guide d'ondes $[6,7,8]:$ l'énergie lumineuse, issue d'un laser est transférée dans le guide à l'aide de dispositifs empruntés à l'optique intégrée $[9,10]$. La deuxième méthode que nous décrirons, consiste à provoquer la

(*) Laboratoire associé au C. N. R. S. réflexion totale sur une structure multicouche dans laquelle la couche mince à étudier agit comme une cavité résonante. La dernière méthode utilise l'excitation des plasmons de surface sur l'interface plane séparant un métal d'un milieu diélectrique. Dans ce dernier cas, le flux Raman diffusé est dû à l'interaction entre l'onde évanescente qui se propage sur l'interface et le matériau diélectrique.

2. Méthodes du guide d'ondes. - 2.1 PRINCIPE. Considérons le guide d'ondes représenté sur la figure 1 ,

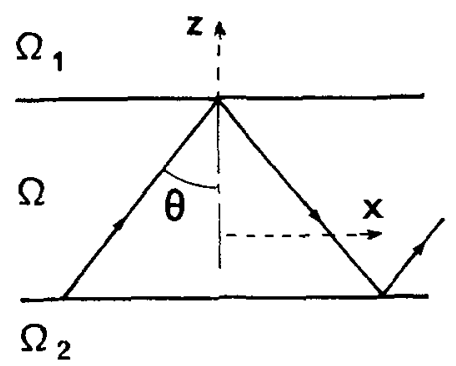

FIG. 1. - Propagation dans un guide d'onde d'indide $n$, d'épaisseur $d, \theta$ est l'angle de réflexion. $\psi_{1}, \psi_{2}$ sont les déphasages à la réflexion totale sur les dioptres $\left(n, n_{1}\right)$ et $\left(n, n_{2}\right)$.

caractérisé par son indice de réfraction réel $n$, séparant l'espace en deux milieux semi infinis d'indices $n_{1}$ et $n_{2}$. Nous supposerons que $n_{2}>n_{1}$. Un tel guide est réalisé dans la pratique en déposant sur un substrat d'indice de réfraction $n_{2}$, une couche mince d'épaisseur $d$. Pour que l'énergie se propage dans la direction $\mathrm{O} x$, sans pertes, il est nécessaire que les ondes dans le guide subissent la réflexion totale sur les deux interfaces limitant la couche. A l'intérieur du guide, le champ électromagnétique résulte de la superposition de deux 
ondes planes d'amplitudes $A$ et $B$. La dépendance en $\exp (j \omega t)$ est implicite.

$$
\begin{aligned}
U(x, z)=\exp (-j k \alpha x) \times & \times[A \exp (-j k \gamma z)+B \exp (+j k \gamma z)]
\end{aligned}
$$

A l'extérieur du guide le champ est évanescent :

$$
\begin{gathered}
U_{1}(x, z)=A_{1} \exp (-j k \alpha x) \exp \left[+k \gamma_{1}(z+d / 2)\right] ; \\
z \leqslant-d / 2 \\
U_{2}(x, z)=A_{2} \exp (-j k \alpha x) \exp \left[-k \gamma_{2}(z-d / 2)\right] ; \\
z \geqslant+d / 2
\end{gathered}
$$

avec $k=2 \pi / \lambda_{0}: \lambda_{0}$ est la longueur d'onde dans le vide.

$\alpha=n \sin \theta$. On a de plus les relations :

$$
\begin{gathered}
\alpha^{2}+\gamma^{2}=n^{2} \\
\alpha^{2}-\gamma_{1}^{2}=n_{1}^{2} \\
\alpha^{2}-\gamma_{2}^{2}=n_{2}^{2} .
\end{gathered}
$$

L'expression $U_{i}(x, z)$ caractérise la composante $E_{y}$ dans le cas T. E. et la composante $H_{y}(x, z)$ dans le cas T. $M$.

On démontre, à partir de ces relations, qu'il se propage dans le guide un ou plusieurs modes de propagation T. E. et T. M. Ces modes non rayonnants sont définis par l'équation suivante :

$2 k n d \cos \theta-\psi_{1}-\psi_{2}=2 m \pi ; \quad m=0,1,2, \ldots$

où $\psi_{1}$ et $\psi_{2}$ caractérisent les déphasages que prennent les ondes $A$ et $B$ lors de la réfiexion totale sur les deux interfaces limitant le guide. $m$ est un entier positif ou nul qui caractérise l'ordre du mode. Les déphasages $\psi_{1}$ et $\psi_{2}$ dépendent de l'état de polarisation T. E. ou T. M. et sont donnés par les expressions

$$
\operatorname{tg} \psi_{i}^{\mathrm{TE}} / 2=\frac{\gamma i}{\gamma} \quad \operatorname{tg} \psi_{i}^{\mathrm{TM}} / 2=\frac{n^{2}}{n_{i}^{2}} \frac{\gamma i}{\gamma} ; \quad i=1,2 .
$$

Afin d'avoir quelques informations pratiques sur la propagation dans le guide, on peut tirer à partir de l'expression (1), l'évolution de la constante de propagation $\alpha=n \sin \theta$ en fonction de l'épaisseur $d$.

La figure 2 caractérise différentes courbes pour diffé-

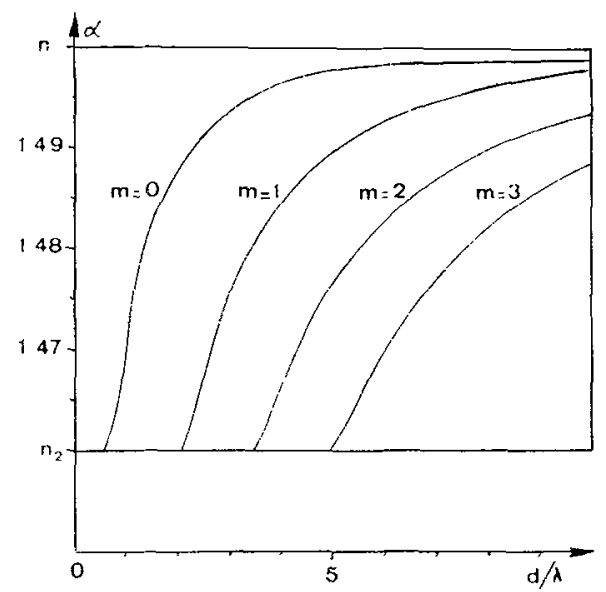

FIG. 2. - Variations de $\alpha=n \sin \theta$ en fonction de $d / \lambda$ pour différents modes de propagation T. E. rentes valeurs de l'entier $m=0,1,2,3,4$, dans le cas de la polarisation T. E. et pour les valeurs $n=1,50$; $n_{1}=1 ; n_{2}=1,46$. Si l'on se place sur la courbe $m=0$ par exemple, on constate que si $d$ diminue, $\alpha$ tend vers l'indice $n_{2}$ du support, ce qui indique que l'énergie pénètre de plus en plus profondément dans le support et à la limite lorsque $\alpha=n_{2}$. l'onde dans le support est homogène. Au contraire, lorsque $d$ augmente, $\alpha$ tend vers $n$ et l'énergie se confine dans le guide. A la limite, le champ évanescent à l'extérieur du guide s'annule. Ainsi la diminution de l'épaisseur $d$ permet de faire basculer l'énergie lumineuse de l'intérieur du guide vers l'extérieur. Il est alors possible d'obtenir séparément ou simultanément le spectre Raman du guide ou de son support. Nous aurons l'occasion dans la partie expérimentale de revenir sur ce point particulier.

2.2 Excitation DU Guide D'ondes. - Pour pouvoir exciter l'un des modes propagation du guide d'ondes, on effectue le couplage de la lumière émise par un laser, par l'intermédiaire d'un prisme [9]. Ce dispositif est représenté sur la figure 3 . Ce prisme est rapproché

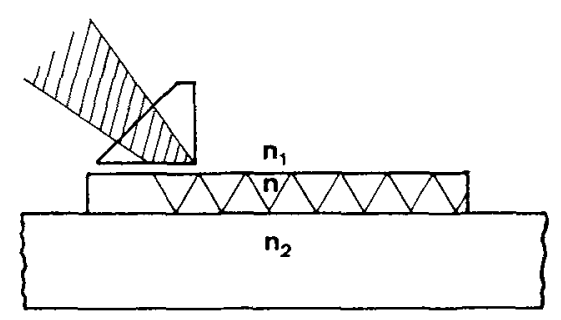

FIG. 3. - Schéma d'un coupleur à prisme. La lumière issue d'un laser est introduite dans le guide $n$ par l'intermédiaire du prisme.

$\mathrm{du}$ guide à une distance optimale. Le faisceau laser tombe au voisinage de l'arête du prisme, sous une incidence très voisine de celle correspondant au mode de propagation du guide, avec la polarisation du mode (T. E. ou T. M.). Si l'étendue du faisceau lumineux est bien adaptée, une fraction importante de l'énergie lumineuse pénètre à l'intérieur du guide et se propage librement au-delà de l'arête vive du prisme. Désignons par $D$ le diamètre du faisceau lumineux. Projetons sur le guide la fente d'un spectromètre dont les dimensions sont égales à $L$ dans la direction de propagation et $D$ dans la direction perpendiculaire. Dans ces conditions on montre que le flux Raman diffusé par le volume $v=d L D$ est beaucoup plus important que celui obtenu par une technique de rétrodiffusion. Dans la méthode du guide d'ondes le flux diffusé est égal à [1] :

$$
\varphi_{\mathrm{G}}=K W L D d
$$

où $K$ est une constante caractéristique du matériau, et $W$ est la densité d'énergie à l'intérieur du guide.

De plus on a la relation :

$$
W=\tau n W_{\mathrm{i}} D / d
$$


où $\tau$ caractérise le coefficient de couplage, c'est-à-dire la fraction d'énergie qui se propage réellement à l'intérieur du guide et $W_{i}$ est la densité d'énergie du faisceau incident dans l'air.

Dans la technique de rétrodiffusion, le flux Raman diffusé avec un faisceau incident de même étendue est égal à :

$$
\varphi_{\mathrm{RD}}=K d D^{2} W_{\mathrm{c}} .
$$

où $d D^{2}$ est le volume utile de diffusion utilisé et $W_{\mathrm{c}}$ est la densité d'énergie dans la couche. Remarquons que $W_{\mathrm{c}}=n W_{\mathrm{i}}$. En effectuant le rapport $R=\varphi_{\mathrm{G}} / \varphi_{\mathrm{RD}}$, on obtient :

$$
R=\tau L / d .
$$

Avec les paramètres expérimentaux suivants:
$L=6 \mathrm{~mm} ; d=1 \mu$ et $\tau=0,3$, le rapport $R$ est voisin de 2000 . L'importance de ce rapport confirme l'intérêt de cette méthode vis-à-vis de la technique de rétrodiffusion.

2.3 RÉSUlTATS EXPÉRIMENTAUX. - Les résultats expérimentaux que nous avons obtenus sont présentés sur la figure 4. Les spectres Raman de deux couches minces de polyméthacrylate de méthyle d'épaisseurs $6 \mu$ et $1 \mu$ sont présentés sur les figures $4 a$ et $4 b$. La figure $4 c$ caractérise le spectre massif du même matériau. On doit noter la différence importante que l'on observe entre les figures $4 a$ et $4 b$, plus particulièrement dans la région des $500 \mathrm{~cm}^{-1}$ où la couche mince de $1 \mu$ est excitée sur un mode propagation pour lequel $\alpha$ est voisin de l'indice du support. Comme nous
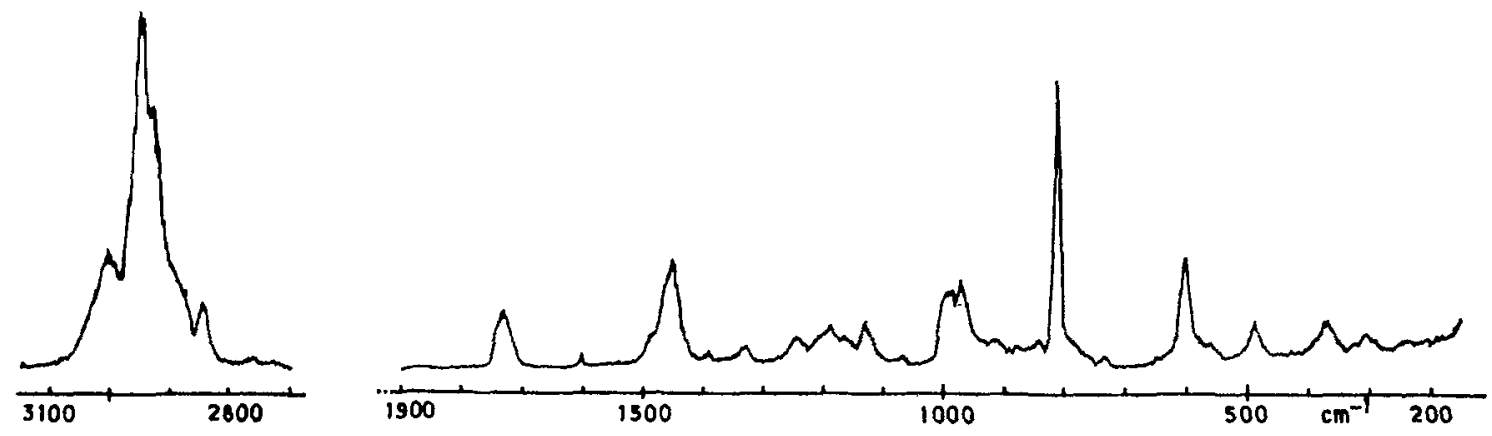

FIG. 4a. - Spectre Raman d'une couche de polyméthacrylate de méthyle de 6 microns d'épaisseur.
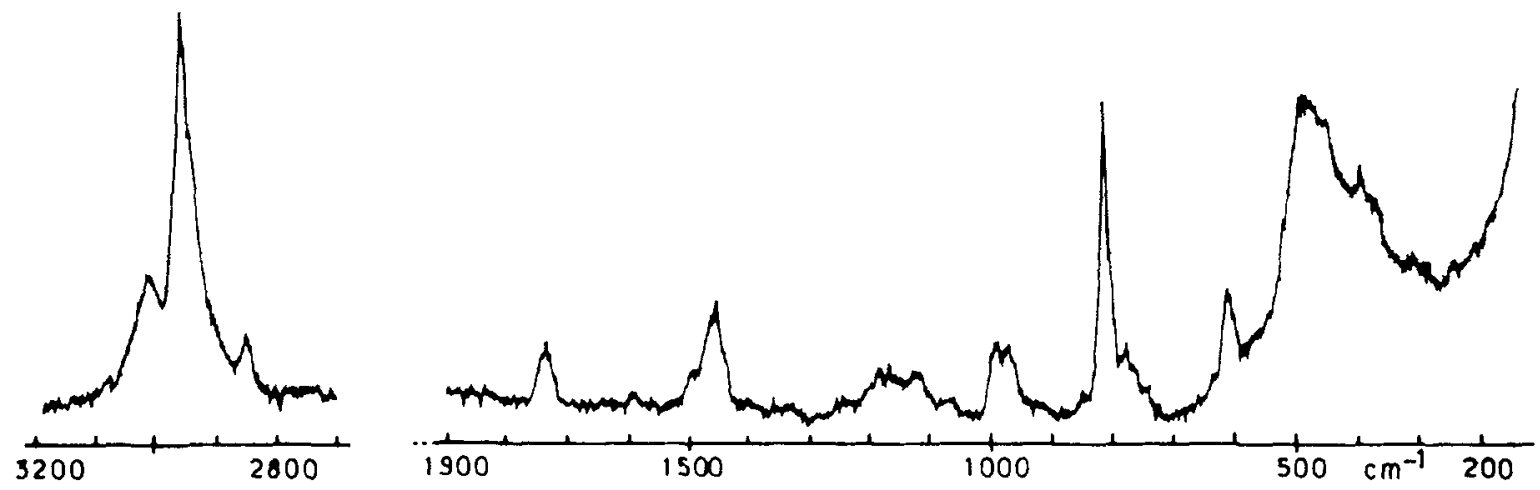

FiG. 4b. - Spectre Raman d'une couche de 1 micron du même matériau. On observe dans la région de $300 \mathrm{~cm}^{-1}-500 \mathrm{~cm}^{-1}$ l'apparition d'une bande due au support.
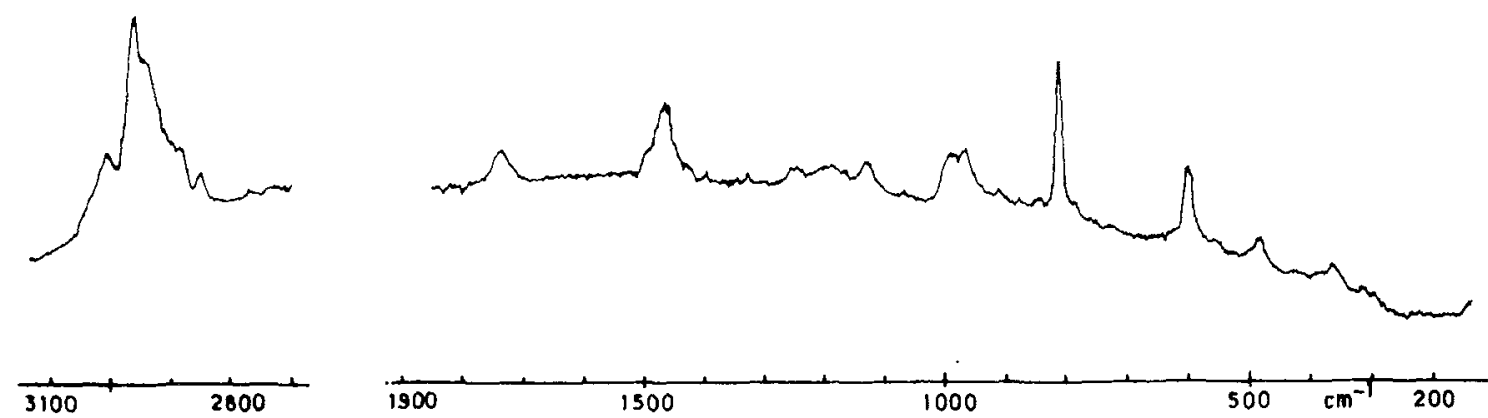

FIG. 4c. - Spectre Raman du matériau massif. Le fond parasite est dû probablement à la présence d'impuretés. 
l'avions indiqué plus haut cela correspond à la situation pour laquelle l'énergie transite suffisamment dans le substrat pour en donner un spectre Raman aisément détectable. C'est précisément ce que l'on observe dans la région des $500 \mathrm{~cm}^{-1}$. Les spectres ont été obtenus avec un double monochromateur. Un laser à argon a été utilisé à $5145 \AA$ avec une puissance de $500 \mathrm{~mW}$ et une polarisation T.E. (perpendiculaire au plan d'incidence).

Remarquons enfin que l'épaisseur du guide ne peut descendre en deçà d'une épaisseur limite voisine de $0,56 \lambda$ (Fig. 2). Pour des épaisseurs plus faibles, l'énergie ne peut plus se propager librement dans le guide. Cette épaisseur critique caractérise la limite de la méthode, pour les valeurs des indices de réfraction choisis dans cet exemple.

3. Réflexion totale sur une structure multicouche. 3.1 Structure DiéleCtriQue. - Considérons la structure à deux couches minces, représentée sur la figure 5: quatre milieux diélectriques, homogènes,

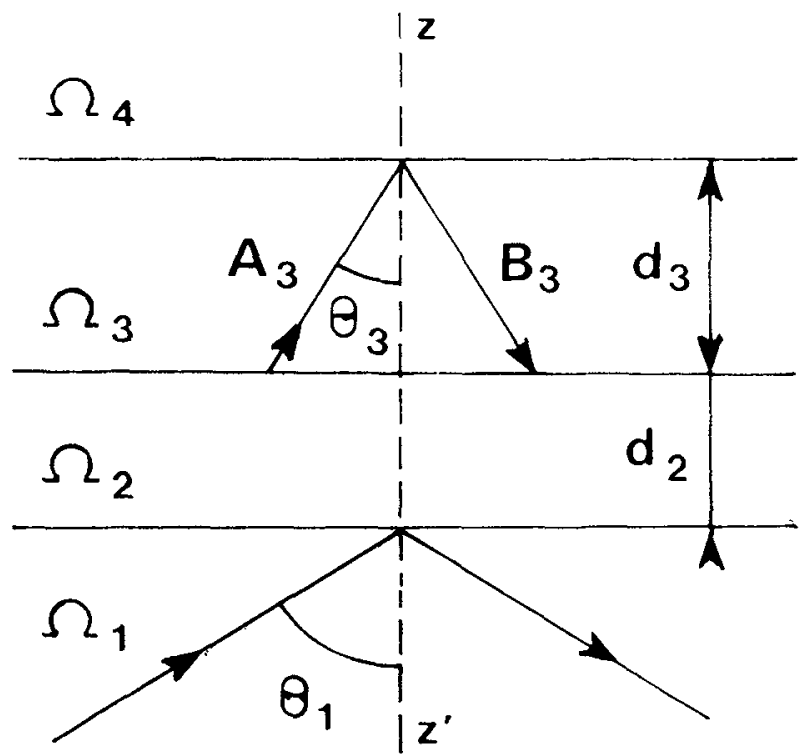

FIg. 5. - Structure multicouche à quatre milieux $-\theta_{1}$ est l'angle d'incidence dans $\Omega_{1}$ et $\theta_{3}$ est l'angle de réfraction dans $\Omega_{3}$.

isotropes et non absorbants, sont séparés par des interfaces planes et parallèles entre elles. Une onde plane monochromatique issue dans le milieu $\Omega_{1}$ tombe sous le dioptre plan $\Omega_{1} / \Omega_{2}$ sous un angle d'incidence $\theta_{1}$. Nous supposerons que la distribution des indices de réfraction du système est telle que les ondes électromagnétiques sont inhomogènes dans $\Omega_{2}$ et $\Omega_{4}$ et homogènes dans $\Omega_{3}$. Ces conditions sont obtenues facilement si l'angle d'incidence $\theta_{1}$ est supérieur aux angles limites définis entre les milieux $\Omega_{1}$ et $\Omega_{2}$ ou $\Omega_{4}$. Ainsi le champ électromagnétique dans $\Omega_{3}$ résulte de la superposition de deux ondes planes homogènes, d'amplitudes $A_{3}$ et $B_{3}$, tour à tour incidentes et réfléchies sur les deux interfaces $\Omega_{3} / \Omega_{4}$ et $\Omega_{3} / \Omega_{2}$. Dans $\Omega_{4}$, l'onde est évanescente et le champ dans $\Omega_{2}$ est dû à la superposition de deux ondes planes évanescentes. Dans ces conditions, le champ électrique $E_{y}$ dans le mode T. E. ou le champ magnétique $H_{y}$ dans le mode T. M. s'écrit dans le milieu $\boldsymbol{\Omega}_{i}$ sous la forme :

$$
\begin{aligned}
& U_{i}(x, z)= \exp (-j k \alpha x) \times \\
& \times\left[A_{i} \exp \left(-j k \gamma_{i} z\right)+B_{i} \exp \left(+j k \omega_{i} z\right)\right] ; \\
& i=1,3
\end{aligned}
$$

avec

$$
\alpha^{2}+\gamma_{i}^{2}-n_{i}^{2} .
$$

$U_{i}(x, z)=\exp (-j k \alpha x) \times$

$$
\begin{gathered}
\times\left[A_{i} \exp \left(-p_{i} z\right)+\left(1-\delta_{i, 4}\right) B_{i} \exp \left(+p_{i} z\right)\right] ; \\
i=2,4
\end{gathered}
$$

avec

$$
\alpha^{2}-p_{i}^{2}=n_{i}^{2} .
$$

$\delta_{m, n}$ est le symbole de Kronecker.

La couche mince $\Omega_{3}$ est utilisée ici comme une cavité résonnante dans laquelle la réflexion est totale sur le dioptre $\Omega_{3} / \Omega_{4}$ et quasi totale sur l'interface $\Omega_{3} / \Omega_{2}$. Lorsque l'épaisseur du milieu $\Omega_{2}$ tend vers l'infini, on retrouve le dispositif du guide d'ondes précédemment décrit. Du fait de sa propriété de cavité résonnante, on démontre que la densité d'énergie dans $\Omega_{3}$ peut atteindre des valeurs très élevées si la condition suivante est réalisée [2] :

$$
2 k n_{3} d_{3} \cos \theta_{3}=\psi_{31}+\psi_{34}+2 m \pi
$$

où $\psi_{34}$ et $\psi_{31}$ caractérisent les déphasages pris par les ondes $A_{3}$ et $B_{3}$ qui se réfléchissent sur les interfaces limitant la cavité résonnante. Il faut noter toutefois que le terme $\psi_{31}$ tient compte de la présence du milieu $\Omega_{1}$. Lorsque $d_{2}$ tend vers l'infini $\psi_{31}$ tend vers la valeur $\psi_{32}$ précédemment définie dans le cas du guide d'ondes.

Parallèlement au phénomène d'accumulation d'énergie dans la cavité résonnante, il a été montré que le champ électromagnétique sur l'interface $\Omega_{3} / \Omega_{4}$ pouvait prendre des valeurs très élevées. Cette importante densité d'énergie est très favorable à l'excitation du spectre Raman de la cavité. La figure 6 caractérise

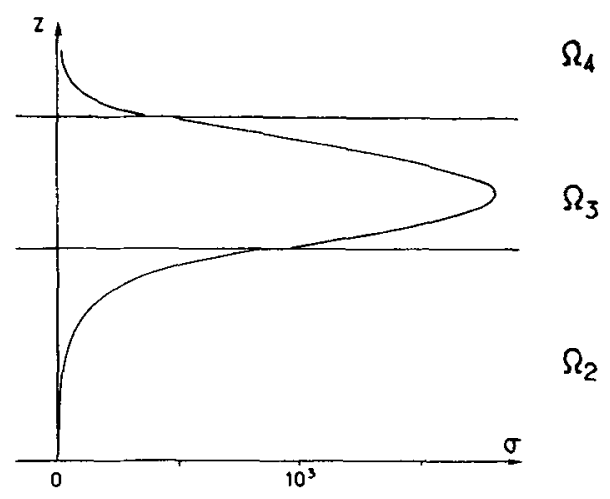

FIG. 6. - Distribution de la densité d'énergie normalisée dans les milieux $\Omega_{2}, \Omega_{3}, \Omega_{4}$ en fonction de la coordonnée $z$. $\sigma$ est le rapport entre la densité d'énergie $W(z)$ dans la structure et la densité d'énergie $W_{1}$ du faisceau incident. $n_{1}=1,70 ; n_{2}=1,30$; $n_{3}=1,48 ; n_{4}=1 ; \theta_{1}=55^{\circ} ; d_{2}=\lambda ; d_{3}=0,60 \lambda$. 
l'évolution de la densité d'énergie $W$ dans la structure rapportée à la densité dans le premier milieu $\Omega_{1}$, en fonction de la coordonnée spatiale $z$. On constate que le maximum de la densité se trouve à l'intérieur de la cavité et atteint une valeur proche de 1600 . A l'extérieur de la cavité, la densité d'énergie décroît exponentiellement. Un calcul simple a permis de montrer que le flux Raman diffusé par la cavité, pour les valeurs indiquées dans la légende de la figure 6 , est environ 3000 fois plus grand que celui obtenu par la méthode de rétrodiffusion [2]. Si l'on compare les résultats de cette méthode à ceux obtenus par le guide d'ondes, on constate que les flux Raman diffusés sont du même ordre de grandeur. Mais le volume de matériau utilisé dans la méthode du guide d'ondes est nettement plus important.

3.2 RÉSULTATS EXPÉRIMENTAUX. - L'étude a porté sur les couches de stéarate de baryum déposées par la méthode de Langmuir-Blodgett $[11,12]$, sur un prisme d'indice de réfraction $n_{1}=1,70$. L'épaisseur des couches est déterminée à mieux que $25 \AA$ et l'indice de réfraction obtenu pour la cavité est égal à 1,48. Les couches de bas indice $n_{2}$ sont réalisées dans les mêmes conditions et sont soumises à un traitement qui crée des lacunes et qui permet d'obtenir un indice de réfraction compris entre 1,20 et 1,50 . La couche réalisée présentait un indice de 1,30. La figure 7 représente le dis-

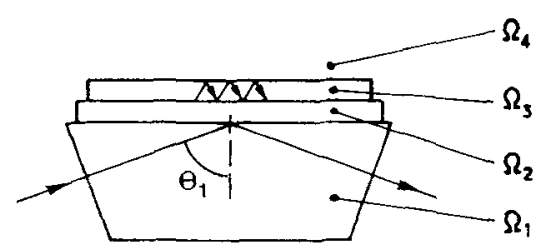

FIG. 7. - Dispositif expérimental représentant le schéma théorique de la figure 5.

positif expérimental avec les valeurs indiquées dans la légende de la figure 6. Les spectres Raman ont pu être obtenus pour les deux états de polarisation T. E. et T. M., dans les conditions indiquées par l'éq. (2). On passe de l'excitation optimale T. E. à l'excitation T. M. en changeant légèrement l'angle d'incidence. Les spectres de la vibration $\mathrm{C}-\mathrm{H}$ ont été obtenus avec quatre polarisations $X X, X Y, Z X, Z Y$, à l'aide de la radiation $4579 \AA$ de puissance $400 \mathrm{~mW}$ et pour une largeur de fente de $6 \mathrm{~cm}^{-1}$ (Fig. 8). Cette méthode présente l'inconvénient de laisser passer les bandes de fluorescence dues au prisme ainsi que les raies provenant des milieux extérieurs à la cavité résonnante. Cet inconvénient nous a conduit à élaborer une nouvelle structure que nous allons décrire.

3.3 StrUCture abSORbante. - Considérons la structure multicouche représentée par la figure 5 où $\Omega_{2}$ est une couche mince métallique et $\Omega_{1}, \Omega_{3}, \Omega_{4}$ sont des milieux diélectriques, non absorbants [3]. Comme précédemment, le champ électromagnétique a une expression analogue au cas de la structure diélectrique. Il est facile d'établir le rapport $\tau_{\mathrm{s}}$ entre la densité d'énergie dans $\Omega_{4}$, sur l'interface $\Omega_{3} / \Omega_{4}$ et la densité d'énergie de l'onde incidente dans $\Omega_{1}$. Ce rapport s'écrit [4] :

$$
\tau_{\mathrm{s}}=\frac{P\left|\exp \left(-j k n_{3} d_{3} \omega_{3}\right)\right|^{2}}{\left|1-\mathbf{r}_{31} \mathbf{r}_{34} \exp \left(-j\left(2 k n_{3} d_{3} \omega_{3}-\psi_{31}-\psi_{34}\right)\right)\right|^{2}}
$$

dont la valeur maximale, pour $\omega_{3}$ réel, est égale à :

$$
\tau_{\mathrm{s}}^{\max }=P /\left|1-\mathbf{r}_{31} \mathbf{r}_{34}\right|^{2}
$$

$P$ est une quantité indépendante de $d_{3}, r_{31} \exp \left(-j \psi_{31}\right)$, et $r_{34} \exp \left(-j \psi_{34}\right)$ sont les facteurs de réflexion sur les interfaces $\Omega_{3} / \Omega_{4}$ et $\Omega_{3} / \Omega_{2}$.

$$
\omega_{3}=\left(n_{3}^{2}-n_{1}^{2} \sin ^{2} \theta_{1}\right)^{1 / 2} / n_{3}=\cos \theta_{3} .
$$

A partir de $\tau_{s}$, on déduit aisément le rapport entre le carré du champ électrique en un point de la structure et celui de l'onde incidente soit $E^{2} / E_{\mathrm{i}}^{2}$. La courbe représentative est indiquée sur la figure 9 . Elle se compose de trois parties, la première portion pour $0<z<d_{2}$ est à croissance exponentielle, la deuxième partie définie par $d_{2}<z<d_{2}+d_{3}$, présente une évolution sinusoïdale, la dernière section caractérisée par $z>d_{2}+d_{3}$ montre une décroissance exponentielle. Dans le but d'optimiser la diffusion Raman, il est nécessaire de rechercher les conditions qui rendent

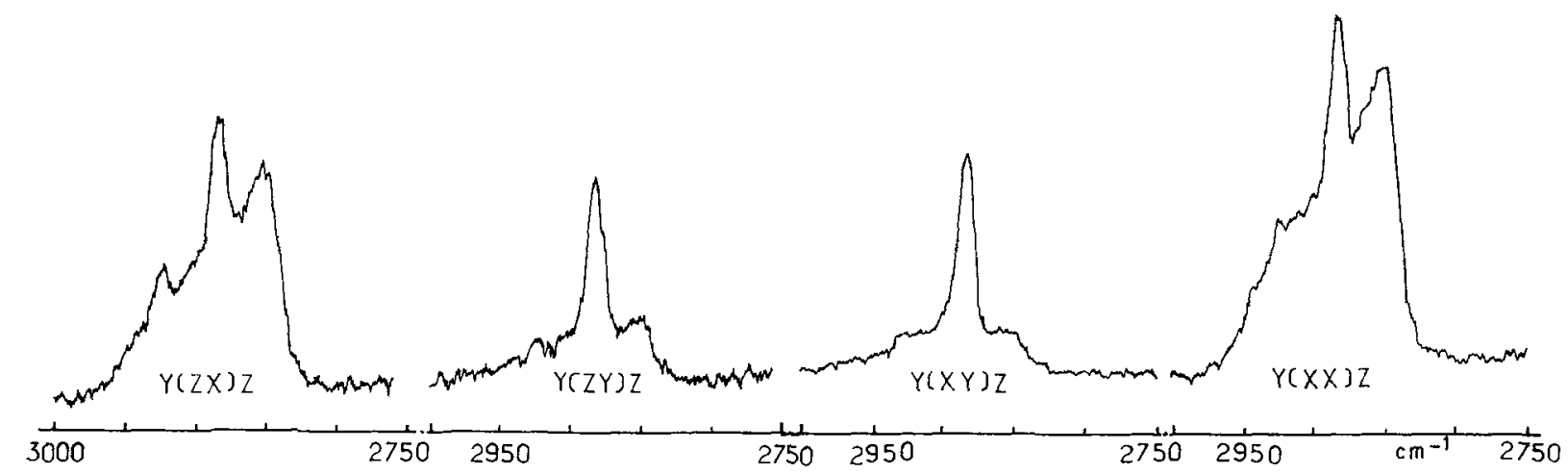

FIG. 8. - Spectre Raman de la bande C-H d'une couche de 0,35 micron d'épaisseur de stéarate de baryum, avec des polarisations différentes. 


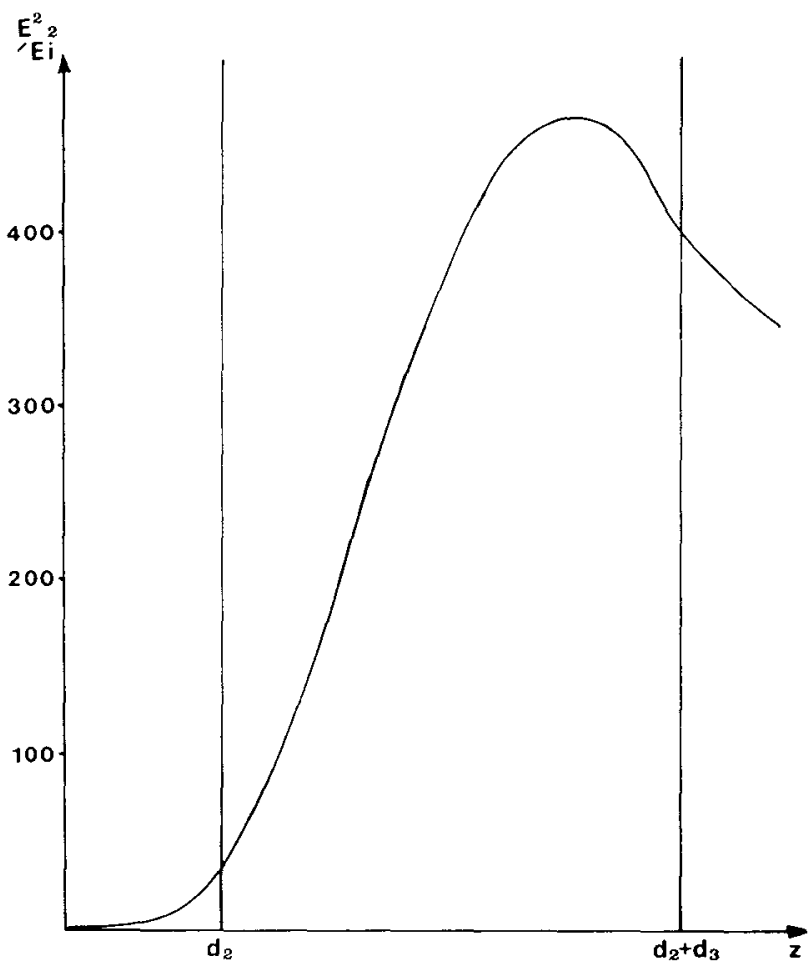

Fig. 9. - Variation du rapport $E^{2} / E_{1}^{2}$ en fonction de la coordonnée $z, E$ est le module du champ électrique en un point de la structure et $E_{\mathrm{i}}$ caractérise le champ de l'onde incidente. Le système est tel que : $\lambda=5500 \AA ; n_{1}=2,9 ; n_{2}=0,084-j 3,67$; $n_{3}=1,5 ; n_{4}=1 ; d_{2}=0,08 \lambda ; d_{3}=0,26 \lambda ; \theta_{1}=22^{\circ}$.

$\tau_{\mathrm{s}}$ maximum. Ce rapport prend une valeur maximale lorsque la condition (2) est réalisée. Pour le mode $m=0$, il existe une solution en $\theta_{1}$ si [13]

$$
\psi_{31}+\psi_{34}>0 \text {. }
$$

Remarquons que le terme $\psi_{31}$ est complexe mais l'on peut négliger sa partie imaginaire dans le cas des métaux que nous considérons. Si le milieu $\Omega_{2}$ est tel que la partie réelle de $n_{2}^{2}$ soit négative (ce qui est le cas des milieux métalliques que nous considérons), cette condition est toujours satisfaite pour le mode TE mais pas nécessairement pour le mode TM.

L'analyse des relations pour le mode $\mathrm{TM}_{0}$ montre que la condition de résonance est satisfaite si $d_{3}=0$. On retrouve alors la configuration proposée par Kreitschmann [14] où la couche métallique sépare deux milieux diélectriques considérés comme semi-infinis. Ce dispositif est celui que l'on utilise pour exciter les plasmons de surface sur l'interface métal $/ \Omega_{4}$. De la même façon, lorsque $d_{3} \rightarrow \infty$ la condition de résonance est encore satisfaite et correspond au dispositif utilisé pour exciter les plasmons de surface entre $\Omega_{2}$ et $\Omega_{3}$. Ce montage particulier a été étudié et utilisé par différents auteurs pour d'autres applications [15].

Lorsque les ondes dans $\Omega_{3}$ deviennent inhomogènes, ce qui se produit lorsque $\sin \theta_{1}>n_{3} / n_{1}$, on a alors $\omega_{3}=-j p_{3}$ et la densité d'énergie normalisée à la surface est de l'ordre de grandeur de

$$
\tau_{\mathrm{s}}^{\prime}=\tau_{\mathrm{s}} \exp \left(2 k n_{3} p_{3} d_{3}\right)
$$

Pour $\theta_{1}$ et $d_{2}$ fixés, $\tau_{\mathrm{s}}$ et $\tau_{\mathrm{s}}^{\prime}$ sont maxima si

$$
\cos \left(\psi_{31}+\psi_{34}\right)=\tau_{31} \tau_{34} \exp \left(-2 k n_{3} p_{3} d_{3}\right) \text {. }
$$

Cette dernière relation jointe à la condition de résonance (2) permet de tracer la courbe de la figure 10

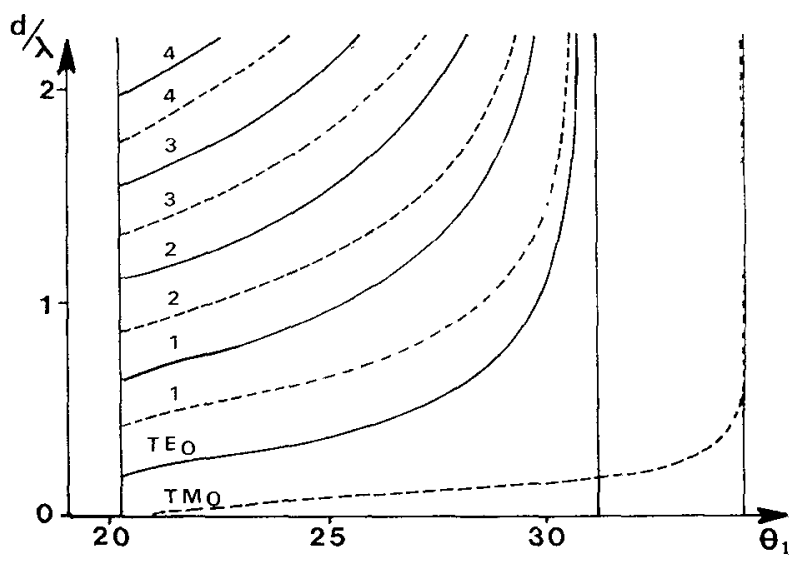

Fra. 10. - Valeurs de l'épaisseur $d_{3}$ en fonction de $\theta_{1}$, donnant lieu à un maximum de $\tau_{\mathrm{s}}$ ou $\tau_{\mathrm{s}}^{\prime}$ pour le système suivant : $n_{1}=2,9$; $n_{2}=0,084-j 3,67 ; n_{3}=1,5 ; n_{4}=1 ; d_{2}=0,08 \lambda$.

qui donne les valeurs de $d_{3}$ en fonction de l'angle d'incidence $\theta_{1}$, pour lesquelles $\tau_{\mathrm{S}}$ ou $\tau_{\mathrm{S}}^{\prime}$ sont maximales. On remarquera encore que $\tau_{\mathrm{s}}$ et $\tau_{\mathrm{s}}^{\prime}$ sont les mêmes pour tous les modes.

L'amplitude de l'onde réfléchie peut s'annuler pour un ensemble de valeurs particulières de $d_{2}, d_{3}, \theta_{1}$ si l'un des milieux est absorbant [16].

Ainsi pour une valeur $d_{2}$ fixée et une polarisation donnée, il est possible d'annuler l'amplitude $B_{1}$ de l'onde réfléchie - ceci correspond à une valeur particulière de l'angle $\theta_{1}$ et un ensemble discret de valeur $d_{3}$ telle que :

$$
d_{3}=d_{3}^{(0)}+\frac{m \lambda}{2 n_{3} \omega_{3}}
$$

Si $d_{3}=0$, il existe encore une valeur $d_{2}$ et un angle d'incidence $\theta_{1}$ pour lequel $B_{1}$ s'annule. L'énergie incidente est totalement absorbée dans le milieu $\Omega_{2}$. Bien que le maximum de $\tau_{\mathrm{S}}$ ou $\tau_{\mathrm{S}}^{\prime}$ ne coïncide pas rigoureusement à l'annulation de $B_{1}$, les deux phénomènes se produisent dans des conditions très voisines (Fig. 11).

3.4 RÉSUltatS EXPÉRIMENTAUX. - On peut utiliser ce système à la spectroscopie Raman de couches minces, du fait de la forte densité d'énergie que l'on peut créer dans la cavité résonnante et dans son voisinage immédiat.

Nous avons étudié le cas d'une couche de polyméthacrylate de méthyle d'épaisseur $2,5 \mu$ déposée sur une couche d'argent, dans deux types d'expériences.

a) la couche mince est utilisée comme cavité résonnante (les ondes $A_{3}$ et $B_{3}$ sont homogènes). L'ensemble de la couche est utilisée sur toute son épaisseur. La figure 12 caractérise l'une des raies obtenue par cette méthode dans les deux cas de polarisation T. E. et 


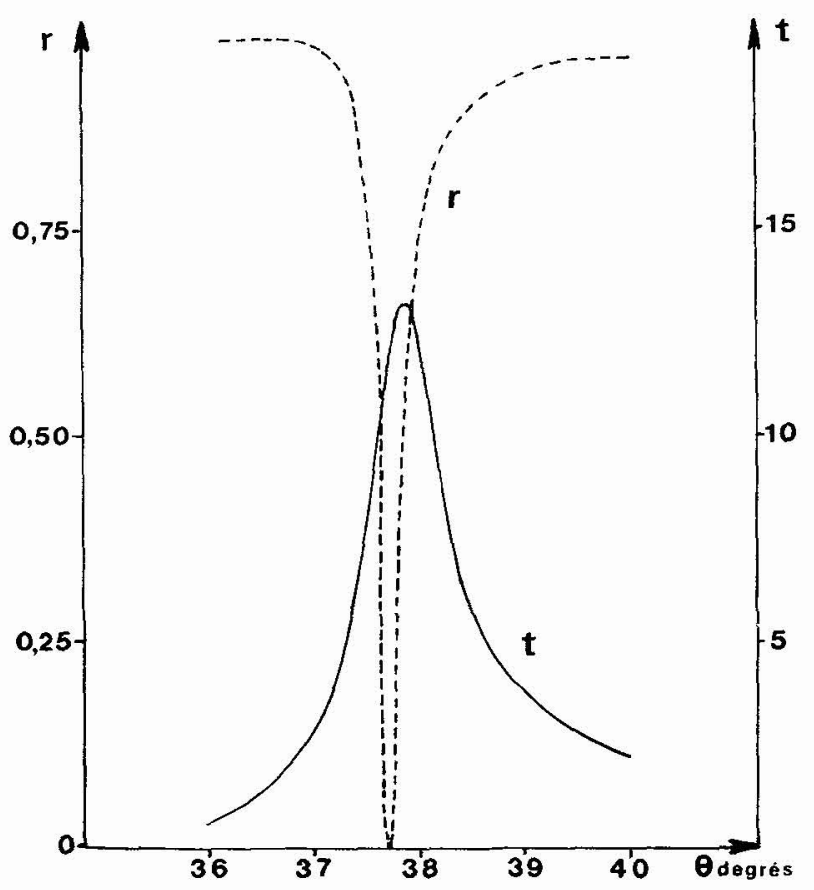

Fig. 11. - Amplitude des facteurs de réflexion $r$ et de transmission $t$ autour de l'angle de résonance $\theta$, pour la polarisation TM $\lambda=5500 \AA ; n_{1}=1,7 ; n_{2}=0,084-j 3,67 ; n_{4}=1$; $d_{2}=0,09 \lambda ; d_{3}=0$.

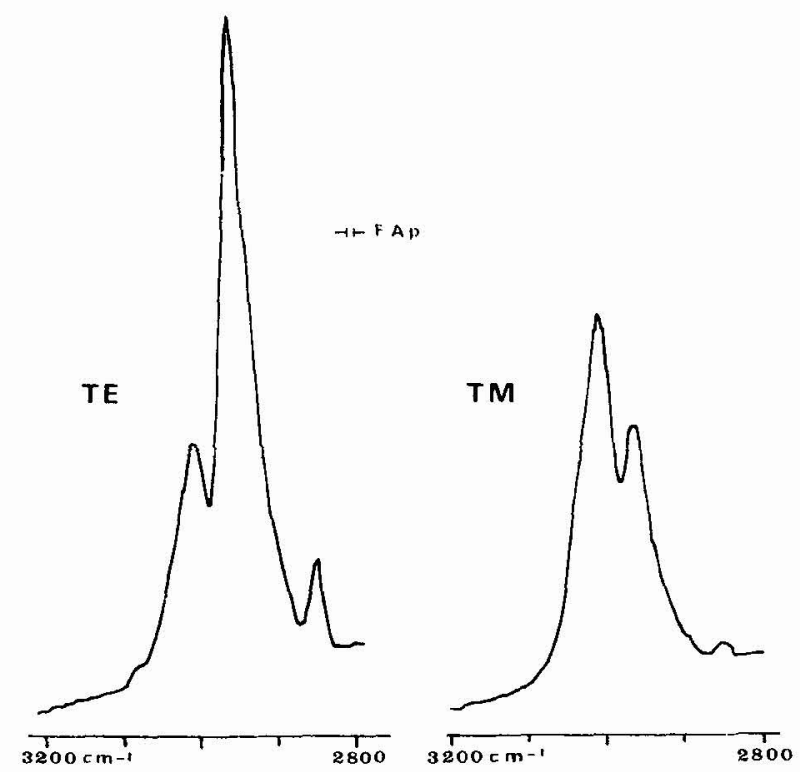

Fig. 12. - Partie du spectre d'une couche de polyméthacrylate de méthyle d'épaisseur 2,5 microns, pour les polarisations TE et TM. La largeur de la fente est de $8 \mathrm{~cm}^{-1}$. La couche est déposée sur une couche d'argent d'épaisseur voisine de $500 \AA$.

T. M. On passe d'un cas de polarisation à l'autre en changeant légèrement l'angle d'incidence.

b) On provoque le phénomène de réflexion totale sur le milieu $\Omega_{3}$ (les ondes $A_{3}$ et $B_{3}$ sont inhomogènes). Il suffit pour cela que $\sin \theta_{1}>n_{3} / n_{1}$. On se place sur le mode T. $\mathrm{M}_{0}, d_{2}$ étant fixée, il existe un angle d'inci- dence pour lequel $\tau_{\mathrm{s}}$ est maximum. La profondeur de pénétration de l'onde évanescente dans $\Omega_{3}$ est égale à :

$$
d_{\mathrm{p}}=\frac{\lambda_{0}}{2 \pi \sqrt{n_{3}^{2}-n_{1}^{2} \sin ^{2} \theta_{1}}} .
$$

$86 \%$ de l'énergie reste confinée à l'intérieur d'une tranche superficielle d'épaisseur $d_{\mathrm{p}}$. Dans le cas de notre exemple $d_{\mathrm{p}} \simeq 1000 \AA$.

$\mathrm{Du}$ fait que l'épaisseur de la couche $(2,5 \mu)$ est très grande devant la profondeur de pénétration $d_{\mathrm{p}}$, le milieu $\Omega_{3}$ peut être considéré comme un milieu semiinfini et l'on retrouve ainsi le cas pratique de l'excitation des plasmons de surface dans la configuration de Kreitschmann.

On peut encore obtenir le spectre Raman d'une mince couche superficielle déposée sur la couche métallique.

Remarquons encore que l'utilisation des plasmons de surface dans l'excitation du spectre Raman, nécessite une vitration incidente T.M. De ce fait, l'onde évanescente dans $\Omega_{3}$ présente une polarisation elliptique. Il est donc nécessaire de tenir compte de cet aspect particulier dans l'interprétation des spectres. La figure 13 montre le spectre obtenu dans ce cas.
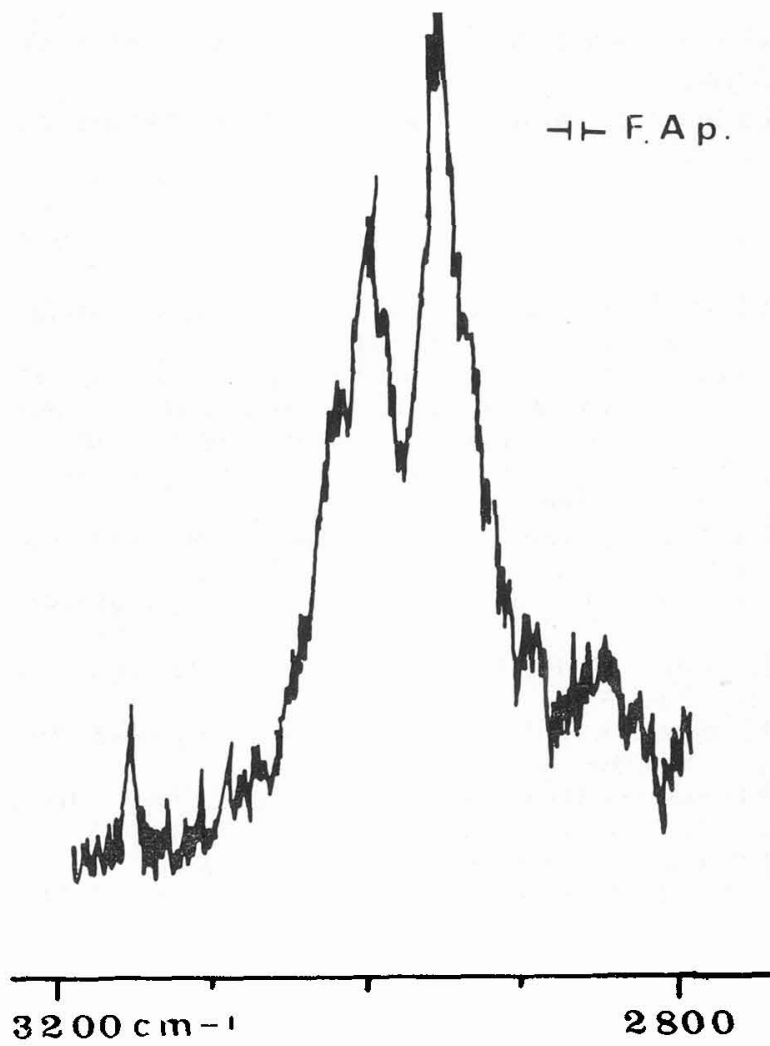

FIG. 13. - Spectre Raman du même matériau excité suivant le mode $\mathrm{TM}_{0}$, par l'intermédiaire de l'onde évanescente à forte amplitude, sur une épaisseur de l'ordre de $1000 \AA$.

On doit remarquer qu'un tel système ne permet pas d'obtenir des densités d'énergie aussi importantes que dans le cas de la structure multicouche diélectrique ; 
en effet si la structure diélectrique permet théoriquement d'atteindre des valeurs aussi élevées que possible, sa limitation pratique est due aux imperfections de surface et inhomogénéités à l'intérieur des couches. Les tolérances sur $d_{2}, d_{3}$ et $\theta_{1}$ deviennent particulièrement critiques lorsque l'on souhaite obtenir des effets de résonance aigus. En d'autres termes, il n'est pas possible d'obtenir dans la cavité des champs électriques aussi intenses que souhaités. Quant à la structure métallique, on doit noter que les coefficients $\tau_{s}$ sont de l'ordre de 20 pour l'or et de 180 pour l'argent dans le cas des valeurs que nous avons utilisées. Mais le facteur de réflexion sous incidence normale, est beaucoup plus élevé que celui de la structure diélectrique. La couche métallique constitue un filtre efficace pour les radiations provenant du support $\Omega_{1}$ et qui peuvent constituer dans certaines régions, un fond parasite plus important que le signal Raman. Les calculs montrent que les couches métalliques transmettent $20 \%$ de l'énergie émise par le support $\Omega_{1}$, dans le cas de l'or et seulement $2 \%$ dans le cas de l'argent.

Un dernier avantage de ce système est que le mode T. $M_{0}$ permet d'obtenir dans $\Omega_{3}$ une onde évanescente à forte amplitude sans nécessiter une couche intermédiaire comme dans le cas des structures diélectriques. Cette couche intermédiaire peut fournir elle aussi quelques raies parasites dans certaines parties des spectres.

La figure 13 caractérise l'une des raies obtenues par excitation des plasmas de surface. Ce spectre est dû à l'interaction de l'onde évanescente qui se propage dans $\Omega_{3}$ sur l'interface $\Omega_{2} / \Omega_{3}$ et d'une tranche superficielle de $\Omega_{3}$ d'épaisseur de l'ordre de $d_{\mathrm{p}}$.

4. Conclusion. - Nous avons présenté les différents systèmes permettant d'obtenir de fortes densités d'énergie à l'intérieur du matériau à étudier et nous avons montré que l'on pouvait déceler le spectre Raman.

Ces différentes méthodes présentent des avantages spécifiques les unes vis-à-vis des autres. La méthode utilisant la couche métallique présente l'avantage d'éliminer la couche intermédiaire indispensable dans la structure diélectrique, d'obtenir une onde évanescente à forte amplitude et d'éliminer partiellement le flux parasite provenant du support. On doit encore remarquer que le spectre des matériaux ont été obtenus avec un spectromètre classique. Il est certain que des systèmes plus élaborés d'acquisition de données et de traitement de signal devraient permettre d'améliorer la qualité des spectres obtenus. De ce fait, il devient possible d'envisager d'appliquer ces systèmes à l'obtention de spectres Raman de couches monomoléculaires adsorbées à l'interface, ou de membranes biologiques. De plus on doit signaler que la dernière méthode est applicable sans aucune difficulté à l'interface métal/ liquide [17] dans les conditions que nous avons précisées plus haut. Des expériences sont en cours et les résultats seront publiés ultérieurement.

\section{Bibliographie}

[1] Levy, Y., Imbert, C., Cipriani, J., RaCine, S. et DupeyRAT, R., Opt. Commun. 11 (1974) 66.

[2] Cipriani, J., Racine, S., Dupeyrat, R., Hasmonay, H., Dupeyrat, M., Levy, Y. et ImBert, C., Opt. Commun. 11 (1974) 70 ; Japan. J. Appl. Phys. 14 (1975) 93.

[3] Menetrier, M., Dupeyrat, R., Levy, Y. et Imbert, C., Opt. Commun. 21 (1977) 162.

[4] Tien, P. K. Ulrich, R., J. Opt. Soc. Am. 60 (1970) 1325 ; LeVY, Y., Nouv. Rev. Opt. Appl. 3 (1972) 25.

[5] Takenara, T. et Nakanaga, T., J. Phys. Chem. 80 (1976) 475.

[6] Wessler, G. R., Rodet, K. et Friese, P., Exp. Tech. Phys. XXI, Heft 2 (1973) 179.

[7] Wessler, G. R., Rodel, K. et Friese, P., Exp. Tech. Phys. XXI, Heft 4 (1973) 343.

[8] Burns, G., Dacol, F., Marinace, J. C., Scott, B. A. et Burstein, E., Appl. Phys. Lett. 22 (1973) 8.

[9] Ulrich, R., J. Opt. Soc. Am. 60 (1970) 1337;

Tien, P. K., Ulrich, R. et Martin, R. J., Appl. Phys. Lett. 14 (1969) 291.
[10] Dakss, M. L., Kunn, L., Heidrich, P. F. et Scott, B. A., Appl. Phys. Lett. 16 (1970) 523;

KogelniK, H. et SosNowski, H., Bell. Syst. Tech. J. 49 (1970) 1602.

[11] Blodgett, K. B. et Langmuir, Y., Phys. Rev. 51 (1937) 964.

[12] Hasmonay, H., Dupeyrat, M. et Dupeyrat, R., $C . R$. Hebd. Séan. Acad. Sci. 277C (1973) 651.

[13] OtTo, A. et SohzER, W., Opt. Commun. 3 (1971) 254.

[14] KretschmanN, E., Z. Phys. 241 (1971) 313.

[15] Abeles, F. et Lopez-Rios, T. dans Polaritons, Proc. 1st Taormina Research Conf. on the structure of Matter, E. Burnstein et F. De Martini eds. (Pergamon Press, New York) 1974, 241 ;

Abeles, F., Thin Solid Films, 34 (1976) 291.

[16] Harrick, N. J. et TurNer, A. F., Appl. Opt. 9 (1970) 2111.

Levy, Y., Nouv. Rev. Opt. Appl. 3 (1972) 25.

[17] Chen, Y. O., Chen, W. P. et Burnstein, E., Bull. Am. Phys. Soc. 21 (1976) 338. 